\title{
A Review of the Detection Methods for Climate Regime Shifts
}

\author{
Qunqun Liu, ${ }^{1,2,3}$ Shiquan Wan, ${ }^{4}$ and Bin $\mathrm{Gu}^{1,2,3}$ \\ ${ }^{1}$ College of Physics, Nanjing University of Information Science and Technology, Nanjing 210044, China \\ ${ }^{2}$ Institute of Space Weather, Nanjing University of Information Science and Technology, Nanjing 210044, China \\ ${ }^{3}$ The Key Laboratory for Aerosol-Cloud-Precipitation of CMA-NUIST, Nanjing 210044, China \\ ${ }^{4}$ Yangzhou Meteorological Office, Yangzhou 225009, China
}

Correspondence should be addressed to Shiquan Wan; wan_sq@163.com

Received 5 November 2015; Accepted 28 December 2015

Academic Editor: Yong-Ping Wu

Copyright (C) 2016 Qunqun Liu et al. This is an open access article distributed under the Creative Commons Attribution License, which permits unrestricted use, distribution, and reproduction in any medium, provided the original work is properly cited.

\begin{abstract}
An abrupt climate change means that the climate system shifts from a steady state to another steady state. Study on the phenomenon and theory of the abrupt climate change is a new research field of modern climatology, and it is of great significance for the prediction of future climate change. The climate regime shift is one of the most common forms of abrupt climate change, which mainly refers to the statistical significant changes on the variable of climate system at one time scale. These detection methods can be roughly divided into five categories based on different types of abrupt changes, namely, abrupt mean value change, abrupt variance change, abrupt frequency change, abrupt probability density change, and the multivariable analysis. The main research progress of abrupt climate change detection methods is reviewed. What is more, some actual applications of those methods in observational data are provided. With the development of nonlinear science, many new methods have been presented for detecting an abrupt dynamic change in recent years, which is useful supplement for the abrupt change detection methods.
\end{abstract}

\section{Introduction}

The research on the phenomenon of abrupt climate change and the related theory is an important field in modern climatology, which is one of the core issues concerning the global climate change. It is also important for the prediction of climate change [1-10]. Since Lorenz [11] and Charney and Devore [12] revealed abrupt climate change on a theoretical basis, the studies on abrupt climate change have been broadly conducted [13-22]. Although the studies on abrupt climate change are relatively late in China, many Chinese climatologists have noticed this phenomenon [23-26]. For example, Chinese scholars first noted the phenomenon of seasonal abrupt change in atmospheric circulation and climate [2734]. Abrupt climate change is a ubiquitous phenomenon in nature. Regarding its physical meaning, it is a transition of the climate system into a different mode on a time scale that is faster than the responsible forcing. Regarding its influence, the occurrence of abrupt change is so rapid and unexpected that human or natural system have difficulty adapting to it
$[35,36]$. These two definitions are complementary: the former provides what abrupt climate change is, and the latter explains why there are so many studies on abrupt climate change.

Because of the suddenness for the occurrence of abrupt climate change, it has a potentially significant impact on the sustainable development of the ecological environment, the social economy, or even the extinction of species. For example, historically, the drought in the region of Mesopotamia led to the decline of ancient civilizations, such as the ancient Greek civilization, the Egyptian civilization in the Nile Valley, and the ancient Indian civilization in the Indus Valley [37]; the Permian-Triassic dinosaur extinction event was also related to abrupt climate change [38]. In 2013, a report by the United States National Research Council noted that when the climate system reaches a certain critical threshold, even a gradual and stable change in the climate system would affect the human infrastructure and the ecosystem. Therefore, the accurate detection and attribution of historical abrupt climate change events have significant practical meaning and potential socioeconomic value for monitoring and forecasting 
the transition or abrupt change that the future climate system may possibly face [39].

At present, there are mainly three aspects of studies on abrupt climate change: early-warning signals for abrupt climate change, detection methods for abrupt climate change, and attribution studies on abrupt climate change events. Scheffer et al. [40] proposed that many complex systems had a critical threshold, that is, a critical point, and that the system would change from one state to another state at this point. This phenomenon is related to the "critical slowing down" phenomenon in the theory of dynamic systems. During the process in which the system approaches the critical point, the critical slowing down phenomenon causes three possible early-warning signals in the dynamical system: a slowing recovery from disturbance, an amplification of autocorrelation, and an increase of variance. There are also numerous studies on the possible reasons that have led to all types of abrupt climate change throughout history. Alley et al. [14] noted that the influence of human activity might increase the probability of the occurrence of abrupt climate change, and once abrupt climate change occurred, it was likely to have a serious potential impact on the economy and the ecosystem. The change in Atlantic thermohaline circulation is likely one reason that caused abrupt climate change [41]. That is, under certain conditions, because of a rapid thawing of ice and melting of snow, more precipitation, and larger discharges from rivers and lakes, much more freshwater is injected into the North Atlantic, therefore causing the salinity and density decrease in the surface water in the North Atlantic. This phenomenon blocks or cuts off the Atlantic thermohaline circulation, causing the temperature to decline rapidly in the surrounding areas of the North Atlantic, leading to abrupt climate change.

The accurate identification and detection of abrupt climate change is the prerequisite and basis for studies on earlywarning signals and attribution studies of abrupt climate change. Many scholars have conducted a lot of relevant studies, such as detection with respect to transition in the climate system state. Rodionov [42] divided these methods into four groups, provided a brief description of each method, as well as their strong and weak points, and pointed out that shifts in the mean are the most common type. Mantua [43] provided a brief review and assessments of the relative strengths and weaknesses of five analytical methods: principal components analysis, vector autoregressive process modeling, autoregressive moving average and intervention analysis modelling, compositing average standard deviates, and Fisher information. Ramanayake and Gupta [44] proposed three statistics, explored their distribution theories, and applied these tests to air traffic data and Stanford Heart Transplant Data. Transition in the climate state, which mainly refers to the notion that there is a statistically significant change in the statistical features of the system state variable over a certain time scale, is one of the most common types of abrupt change phenomenon. This type of abrupt climate change can be roughly divided into five categories: abrupt mean value change, abrupt variance change, abrupt frequency change, abrupt probability density change, and multivariate analysis.
In this paper, we mainly review the progress on the theory and methodological studies in China and other countries regarding the detection of regime shifts in the climate system. The discussions are focused on describing the climate regime shifts detection methods, and some applications on actual observation data. This paper is organised as follows. In Section 2, we briefly introduce the abrupt change detection methods in mean value. Section 3 shows the detection methods for the abrupt probability density change, and the other abrupt change detection methods are presented in Section 4. Discussions and conclusions follow in Section 5.

\section{Abrupt Mean Value Change Detection Methods}

The detection methods for the abrupt mean value changes can be divided into six categories, which mainly include parameter detection method, non-parameter detection method, cumulative sum method, Bayesian analysis method, sequential method, and detection methods based on regression analysis. The moving $t$-test (MTT) $[45,46]$ is a classical parameter detection method, which is a widely applied method in meteorology. It is often used to determine whether abrupt change will occur by testing the difference between the mean values of two sequences. The advantages of MTT include simple procedures, relatively high operational efficiency, and clear physical meaning, that is, strong theoretical basis. However, the assumption of normality needs to be satisfied in using MTT, which makes its scope of application limited. Li et al. [45] presented a successive MTT method and used the MTT method to conduct a comprehensive detection on global abrupt climate change. They found that there was the phenomenon of abrupt climate change in the intensity of atmospheric circulation, South Asian summer monsoon, and global temperatures in the last century. Xiao et al. [16, 18] also employed the successive MTT method to detect the decadal abrupt change in the time series of several atmosphere-ocean variables and found that the warming of the northern midlatitude SST might be responsible for the northern atmospheric decadal abrupt changes and caused the climate change events in the 1980s.

The nonparametric detection methods mainly include the Mann-Kendall test (M-K test) [47, 48], the Mann-Whitney $U$ test [49], the Lepage test [50], and wavelet analysis [51]. The M-K test method presumes that the sequence of elements to be analysed is a stationary time series, which obeys normal distribution, and individual samples are relatively independent. $\mathrm{M}-\mathrm{K}$ method is mainly used to test the trend of abrupt change in a time series. For a time series of $\left\{x_{i}, i=\right.$ $1,2,3, \ldots, N\}$, the cumulative number for the records which are less than $x_{i}$ in subsequences $\left\{x_{j}, j=1,2,3, \ldots, i\right\}$ is represented by $m_{i}$, and the total cumulative number is denoted as follows:

$$
d_{k}=\sum_{i=1}^{k} m_{i}, \quad(2 \leq k \leq N) .
$$


The mean and variance of the newly established sequence $\left\{d_{k}, i=1,2,3, \ldots, N\right\}$ can be, respectively, defined as

$$
\begin{aligned}
E_{\left(d_{k}\right)} & =\frac{k(k+1)}{4}, \\
\operatorname{Var}_{\left(d_{k}\right)} & =\frac{k(k-1)(2 k+5)}{72}, \quad(2 \leq k \leq N) .
\end{aligned}
$$

The standardization of the newly established sequence $\left\{d_{k}\right\}$ can be obtained:

$$
U_{\left(d_{k}\right)}=\frac{\left[d_{k}-E_{\left(d_{k}\right)}\right]}{\sqrt{\operatorname{Var}_{\left(d_{k}\right)}}},
$$

where $U_{\left(d_{k}\right)}$ obeys the standard normal distribution. If the probability density distribution is $\alpha_{1}=\operatorname{prob}\left(|U|>\left|U_{\left(d_{k}\right)}\right|\right)$ for a given significance level $\alpha_{0}$, the original hypothesis is valid for $\alpha_{1}>\alpha_{0}$. The hypothesis is null for $\alpha_{1}<\alpha_{0}$, which indicates that there is a significant trend change in this series. The standardized new sequence $U_{\left(d_{k}\right)}$ consists of a curve of temporal variation. If the curve falls in the confidence interval, there is no significant variation trend in the original sequence; otherwise, the variation trend is significant. The M$\mathrm{K}$ method also has wide application in detecting the abrupt trend change in a time series. For example, $\mathrm{Ma}$ and $\mathrm{Fu}$ [52] used the M-K test to analyse the time-space structure of drywet change in the northern China over the past 54 years and found that there was only one dry-wet conversion on the decadal scale in the eastern area of northwest China and northern China at the end of 1970s. He et al. [53] found that the $\mathrm{M}-\mathrm{K}$ results were completely different for an artificial time series when the $\mathrm{M}-\mathrm{K}$ method was applied to analyse different numbers of samples, which indicated that the $\mathrm{M}$ $\mathrm{K}$ results heavily depend on sample sizes. There were also failures which occurred in the application of MK method in detecting the changes points, such as no change point in an obvious step time series [54].

The Mann-Whitney $U$ test, also called the rank-sum test, was proposed jointly by Mann and Whitney in 1947. This method assumes two samples that derive from two different populations for which all of the statistical characteristics are completely identical except for the mean value. On this basis, it tests whether there is a significant difference in the mean values of these two populations, that is, whether there is an occurrence of abrupt change. The detailed procedures of this algorithm are as follows:

Original hypothesis $H_{0}$ : There is no significant difference between the two samples.

$H_{1}$ : There is a significant difference between the two samples.

Step 1. Combine two sets of data, and assign numeric ranks to all the records, beginning with 1 for the smallest value (where there are groups of tied values, assign a rank equal to the midpoint of unadjusted rankings [e.g., the ranks of $(3,5,5$, 9) are $(1,2.5,2.5,4)])$.
Step 2. Calculate the sum of the ranks for the two samples, and $W_{1}$ and $W_{2}$ are the sums of the ranks in the first sample and another one, respectively.

Step 3. Calculate the statistical quantity $U$ of the MannWhitney $U$ test:

$$
\begin{aligned}
& U_{1}=n_{1} n_{2}+\frac{n_{1}\left(n_{1}+1\right)}{2}-W_{1}, \\
& U_{2}=n_{1} n_{2}+\frac{n_{2}\left(n_{2}+1\right)}{2}-W_{2},
\end{aligned}
$$

where $n_{1}$ is the sample size for the first sample, and $n_{2}$ is the sample size for the second sample. The smaller value of $U_{1}$ and $U_{2}$ is the one used when consulting significance tables and comparing with the given critical value $U_{\alpha}$. If the smaller value of $U_{1}$ and $U_{2}$ is smaller than $U_{\alpha}$, the hypothesis $H_{1}$ is accepted; that is, there is the occurrence of an abrupt change with a significance level $U_{\alpha}$.

If the original hypothesis is true, then the mean value and variance of random variable $U$ are, respectively,

$$
\begin{aligned}
& E(U)=\frac{n_{1} n_{2}}{2}, \\
& D(U)=\frac{n_{1} n_{2}\left(n_{1}+n_{2}+1\right)}{12} .
\end{aligned}
$$

If $n_{1}$ and $n_{2}$ are both not smaller than 10 , the random variable $U$ approximately meets the normal distribution.

Step 4. Make the judgment. Setting the mean value of the first sample as $\mu_{1}$ and the mean value of the second sample as $\mu_{2}$, there are the following:

(1) $H_{0}: \mu_{1} \leq \mu_{2}, H_{1}: \mu_{1}>\mu_{2}$, if there is $U<-U_{\alpha}$, and reject $H_{0}$;

(2) $H_{0}: \mu_{1} \geq \mu_{2}, H_{1}: \mu_{1}<\mu_{2}$, if there is $U>-U_{\alpha}$, and reject $H_{0}$;

(3) $H_{0}: \mu_{1}=\mu_{2}, H_{1}: \mu_{1} \neq \mu_{2}$, if there is $U>-U_{\alpha} / 2$, and reject $H_{0}$.

The Mann-Whitney $U$ test is more widely applicable than independent samples Student's $t$-test, and the question is, which one of them should be preferred.

The Lepage test is a nonparametric statistical method for testing whether there is a significant difference between the mean values of two independent samples, even if the distributions of parent populations are unknown. If there is a significant difference between the two subsequences at a significant level, it is concluded that there is an abrupt change.

Suppose that the number of samples for the subsequence before the reference point is $n_{1}$, the number of samples for the subsequence thereafter is $n_{2}$, and $n_{12}$ is the sum of $n_{1}$ and $n_{2}$. And then, we can calculate the rank sequence $\left\{s_{i}, i=\right.$ $\left.1,2,3, \ldots, n_{12}\right\}$. If the minimum appears before the reference 
point, $s_{i}=1$; otherwise, $s_{i}=0$. We then construct a quantity of rank statistics as $W$ :

$$
W=\sum_{i=1}^{n_{12}} i s_{i}
$$

$W$ is the cumulative number of the two subsequences. Its mean value and variance are, respectively,

$$
\begin{aligned}
E(W) & =\frac{1}{2} n_{1}\left(n_{1}+n_{2}+1\right), \\
\operatorname{Var}(W) & =\frac{1}{12} n_{1} n_{2}\left(n_{1}+n_{2}+1\right) .
\end{aligned}
$$

Additionally, we then construct another quantity of rank statistics as $A$ :

$$
A=\sum_{i=1}^{n_{1}} i s_{i}+\sum_{i-n_{1}+1}^{n_{12}}\left(n_{12}-i+1\right) s_{i} .
$$

$A$ is the sum of the cumulative number of the two subsequences. The first half is the cumulative number of the subsequence before the reference point, and the second half is the cumulative number of the subsequence after the reference point. If $n_{12}$ is even, the mean and variance of $A$ are as follows:

$$
\begin{aligned}
E(A) & =\frac{1}{4} n_{1}\left(n_{1}+n_{2}+2\right), \\
\operatorname{Var}(A) & =\frac{n_{1} n_{2}\left(n_{1}+n_{2}-2\right)\left(n_{1}+n_{2}+2\right)}{48\left(n_{1}+n_{2}-1\right)} .
\end{aligned}
$$

Otherwise,

$$
\begin{aligned}
E(A) & =\frac{n_{1}\left(n_{1}+n_{2}+1\right)^{2}}{4\left(n_{1}+n_{2}\right)}, \\
V(A) & =\frac{n_{1} n_{2}\left(n_{1}+n_{2}+1\right)\left[\left(n_{1}+n_{2}\right)^{2}+3\right]}{48\left(n_{1}+n_{2}\right)^{2}} .
\end{aligned}
$$

A combine of the squares of the standardized Wilcoxon and Ansariy-Braley's statistics is the Lepage test:

$$
W A=\frac{[W-E(W)]^{2}}{\operatorname{Var}(W)}+\frac{[A-E(A)]^{2}}{\operatorname{Var}(A)} .
$$

The Lepage test has more powerful functions than the moving $t$-test and the rank-sum test. Not only can it be used to test abrupt changes but it can also be applied to study the linear trend and periodic variation of climate change. Yonetani $[55,56]$ provided a detailed description of the procedures for using the Lepage method in climate change and applied this method to test abrupt climate change in Japan since 1900. The wavelet analysis is also another abrupt change detection method based on the non-parametric regression function. Antoniadis and Gijbels [57] applied the wavelet analysis method to detect abrupt change and found that this method could well detect an abrupt change in the discharge of the Nile River that occurred in 1898.
The cumulative sum method is the third type of detection method for the abrupt mean value change, and it mainly includes the cumulative sum test and the cumulative frequency test. The cumulative sum method was proposed by Page [58] and then it was subsequently continuously improved [59-61]. The main idea of this method is to obtain the evolutionary trend of a time series by calculating the cumulative sum of the differences between the values and the average in the time series, and then we can identify whether there is a significant trend change in the time series. If the values are all above average during a period of time, the amounts added to the cumulative sum will be positive and the sum will steadily increase, and vice versa. The algorithm of this method is relatively simple, and the cumulative anomaly, $C_{d}(t)$, for a time series can be obtained as follows:

$$
C_{d}(t)=\sum_{i=1}^{t} x_{d i}
$$

where $x_{d i}$ is the sample anomaly. When the absolute value of $C_{d}(t)$ reaches the maximum, the corresponding time is likely the time for the occurrence of the abrupt change. Janssen [62] applied the model to test the cumulative sum method and used this method to test the change in GPS data. Elsner et al. [63] used the cumulative frequency method to test the occurrence frequency of hurricanes in two areas where hurricane often happens and found that there was a significant change in the frequency of hurricanes. The result was consistent with climatic warming in North Africa.

The Bayesian analysis is the fourth detection method type for the abrupt mean value change, which is developed based on Bayesian theory. Firstly, we can integrate the a priori information and sample information of unknown parameters and then derive the a posteriori information according to Bayesian theory. The unknown parameters can be inferred according to the derived posteriori information. The Markov Chain Monte Carlo (MCMC) approach [64] is one of Bayesian analysis. MCMC was developed in the early 1950s. Within the framework of Bayesian theory, the Markov process was introduced into the Monte Carlo simulation, which compensated for the defect in traditional Monte Carlo integration; that is, it only can be used to conduct a static simulation. The procedures of the MCMC algorithm are shown as follows.

Step 1. Construct the Markov chain and make it converge with the stationary distribution.

Step 2. Draw the sample from a target space, apply the chain constructed in Step 1 to sample and simulate, and then generate the sequence.

Step 3. Conduct the Monte Carlo integration, and the expected estimate of any function is as follows:

$$
E[f(x)]=\frac{1}{n-m} \sum_{t=m+1}^{n} f\left(x^{(t)}\right) .
$$




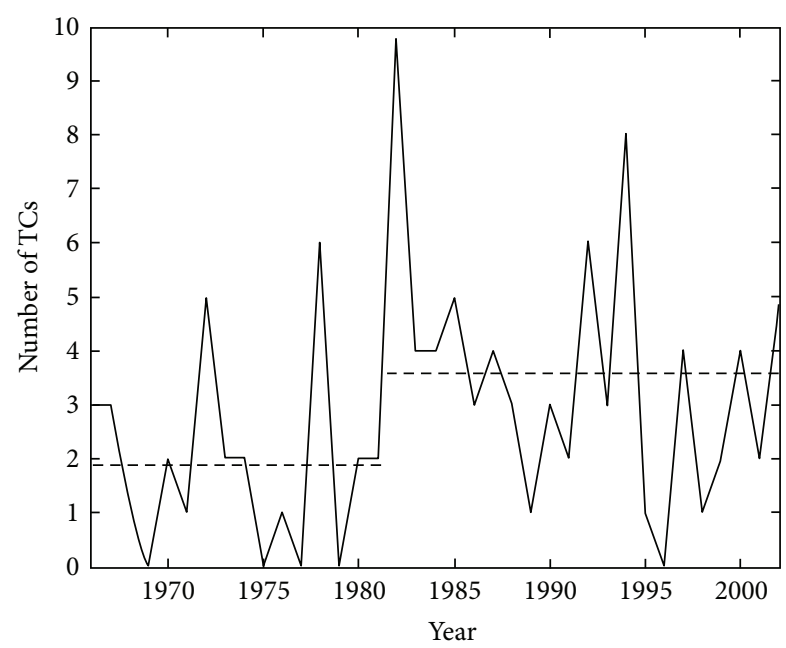

FIgURE 1: The time series of tropical cyclones number in the central of North Pacific during 1966-2002 and its MCMC detection results [65].

Chu and Zhao [65] used the Bayesian analysis to test the variation in the annual number of tropical cyclones in the central North Pacific during 1966-2002 and found that it was extremely possible that there was an abrupt mean change in the number of tropical cyclones in 1982 (Figure 1). Elsner et al. [66] applied MCMC to test the variation in the annual number of hurricanes in North Africa during the 20th century and disclosed that there were three abrupt climate changes in the annual number of hurricanes in the middle of the 1940s, the 1960s, and 1995, respectively.

The sequential method can find the maximum of the mean value from a number of normal distributions with different mean values and then can be used to conveniently and rapidly detect an abrupt mean change. Rodionov [67] noted that the sequential method was better than MTT. In using the sequential method, it is not necessary for making an a priori assumption for a time series and can be used to detect the change point in advance. The sequential $t$-test analysis method (STARS) has several advantages, including analysing the real-time data, timely monitoring of the signal of the change point, and the evolutionary trend of the signal strength. STARS does not need to make an a priori assumption on the change point in a time series and also can be used to deal with the time series with multiple change points. Rodionov and Overland [68] used STARS to test the marine ecosystem index of the Bering Strait and found that the climate transition in 1977 was the strongest in all sea-air index records related to the Bering Strait since the beginning of the 20th century. Reid et al. [69] had employed the Rodionov method to perform a comprehensive detecting on the 1980s regime shift. The shift in temperature that occurred at this time is hypothesized as the main forcing for a cascade of abrupt environmental changes.

Bernaola-Galvan et al. [70] introduced a heuristic segmentation algorithm to probe the temporal organization of heterogeneities in human heartbeat interval time series.
They found that the lengths of segments with different local mean heart rates followed a power-law distribution. Feng et al. [71] verified the performances of the segmentation algorithm in dealing with nonlinear time series by using a number of artificial time series and found that the method could be effectively used to detect an abrupt mean change. Gong et al. [72] used the heuristic segmentation algorithm to analyse northern hemisphere tree rings and Beijing stalagmite based on high and low frequency series and distinguished abrupt changes in different scales and disclosed its physical mechanism.

The two-phase regression technique (TPR) is a typical method for detecting abrupt change based on regression analysis. Hinkley [73, 74] proposed the TPR model and presumed that the regression response is continuous at the point of abrupt change. This assumption is unrealistic, and the influence of the actual environment and instruments may result in the sequence discontinuous. Lund and Reeves [75] found this problem and made an improvement (i.e., the LR method), but the improved method can cause several false points of abrupt change. Subsequently, Wang [76] also found that although the LR method could make the presumed climatic conditions unrealistic because abrupt change might not affect the mean value and trend simultaneously. In order to deal with this problem, Wang proposed a trend model, and the advantage of this model is that it presumes the presence of a trend in a time series and allows the trend to change at the point of abrupt change.

The composite analysis $[77,78]$ is a detection method for a regime shift, and a composite analysis of climatic records is likely to find them in Gaussian, red noise with stationary statistics. Rudnick and Davis [77] examined the composite analysis using random independent time series generated to have the same frequency content as the Pacific Decadal Oscillation (PDO) and found that it was likely to find ostensibly significant steps even if the input time series were red noise. Rodionov [78] proposed a prewhitening procedure which was designed to remove the red noise component from time series prior to applying a regime shift detection method. The application of this method to analyze PDO shows that PDO appears to be more than just a manifestation of a red noise process. The size of subsamples should be as small as possible to minimize the effect of regime shifts on the parameter in the first order autoregressive model. On the other hand, it should be as large as possible to minimize the sampling variability of the parameter. These opposite requirements to the size of subsamples make it difficult to apply this procedure to short time series with decadal or shorter regimes.

\section{Detection Methods for the Abrupt Probability Density Change}

The methods that detect the change in the probability density distribution of elements include the abrupt density change, skewness and kurtosis coefficient, and sliding skewness index method based on the Box-Cox transformation.

For a stable dynamic system, the probability density of the system variables has a relatively stable distribution pattern, although, when the dynamic structure of the system changes, 
it may cause different degrees of change in the distribution patterns of the system variables. From the perspective of identifying small changes in the probability density distribution of the system variables, Cheng et al. [79] applied the coefficient of skewness and kurtosis, which described the characteristics of the probability distribution for a time series, to detect an abrupt change in time series. The numerical test indicates that the coefficients of skewness and kurtosis have a very good capability to identify an abrupt probability density change in artificial signals. The detection results have a relatively small dependence on the sample sizes.

Skewness is a measure of the asymmetry of the probability distribution of a real-valued random variable about its mean. Coefficient of skewness can be positive or negative, or even undefined. For a time series $\left\{x_{i}, i=1,2,3, \ldots, N\right\}$, coefficient of skewness $g$ is defined as

$$
g=\frac{\sum_{i=1}^{n}\left(x_{i}-\bar{x}\right)^{3}}{(n-1) \sigma^{3}} .
$$

The parameter $\sigma$ is the sample standard deviation. The coefficient of skewness $g=0$ when the probability density curve is normal distribution. For $g<0$, the distribution has a negative deviation and the left tail is longer than the right one, and vice versa. The skewness is not strictly connected with the relationship between the mean and median: a distribution with negative skew can have the mean greater than or less than the median, and likewise for positive skew.

Kurtosis is a descriptor of the shape of a probability distribution and, just as for skewness, there are different ways of quantifying it for a theoretical distribution and corresponding ways of estimating it from a sample from a population. Coefficient of kurtosis is mainly used to describe the steep degree of the probability density distribution curve. The standard measure of kurtosis is based on a scaled version of the fourth moment of the data or population, and the coefficient of kurtosis $k$ is defined as

$$
k=\frac{\sum_{i=1}^{n}\left(x_{i}-\bar{x}\right)^{4}}{(n-1) \sigma^{4}} \text {. }
$$

The coefficient of kurtosis of a normal distribution is $k=3$. For $k>3$, the probability distribution is steep compared to that for normal distribution, and the distribution is flat compared to normal distribution for $k<3$.

It should be noted that when the difference in the steepness or skewness of the probability density curve before and after an abrupt change is relatively small, it might need more samples to identify the abrupt change to ensure the statistical reliability of the detection results. Consequently, under certain conditions, the coefficients of skewness and kurtosis most likely have a relatively high requirement regarding the sample size and are likely not applicable to those time series with relatively a small sample size.

He et al. [80] constructed 1000 groups of daily precipitation records with two change points which generated by a weather generator. In these artificial precipitation data, the probability density distributions are obviously differences before and after the change points. The testing results showed that the coefficients of skewness and kurtosis both cannot detect the abrupt change in most of the 1000 groups of artificial data. The coefficient of skewness can only detect two groups, and the coefficient of kurtosis cannot detect these preset change points. The main causes for this were that the daily rainfall data had very strong skewness and the coefficient of skewness and kurtosis might not be sensitive enough to the changes of the probability density distribution of daily precipitation data. In view of this, He et al. [80] put forward the sliding transformation parameter (STP) based on the Box-Cox transformation. A large amount of numerical experiments showed that STP could identify the abrupt probability density change in the artificial series, and it performed much better than the kurtosis coefficient and the skewness coefficient.

Box-Cox transformation [81] is a mathematical transformation tool which can transform a time series of skewed distribution into a new time series with approximative normal distribution. For a time series $\left\{x_{i}, i=1,2,3, \ldots, N\right\}$, BoxCox transform is defined as

$$
Y_{i}(\lambda)= \begin{cases}\frac{x_{i}^{\lambda}-1}{\lambda}, & \lambda \neq 0 \\ \ln \left(x_{i}\right), & \lambda=0,\end{cases}
$$

where $Y_{i}$ is the response variable by Box-Cox transformation, and $\lambda$ is the transformation parameter. This formula only can be used when observational data $x_{i}>0$. Thus, Box and Cox [82] proposed a modified version which could be used for all kinds of data, and the revised Box-Cox transform is defined as

$$
Y_{i}(\lambda)= \begin{cases}\frac{\left(x_{i}+\delta x\right)^{\lambda}-1}{\lambda}, & \lambda \neq 0 \\ \ln \left(x_{i}+\delta x\right), & \lambda=0\end{cases}
$$

where $\delta x$ is a positive number and $x_{i}+\delta x>0$. The parameter $\lambda$ can be estimated by the maximum likelihood method.

In order to test the performance of the STP method, the 1000 artificial daily precipitation series have been analyzed, and the results indicate that STP can almost recognize all of the first change point and the accuracy for the second change point is $99.8 \%$. It shows the ability of STP to identify abrupt probability density changes in daily precipitation series. To examine the performances of STP in the observed daily precipitation records, $\mathrm{He}$ et al. randomly select six observational stations in China and find that all the STP results for six daily precipitation records display almost identical evolutionary trend. The results indicate that the transformation parameters undergo a rapid shift from one stable state to an alternative stable state with obviously different means between 1979 and 1980 (Figure 2), which can be detected by MTT with a significance level $\alpha=0.01$ with the sample size of 10 years.

Moreover, Ning and Gupta [83] studied the performance of generalized lambda distributions (GLD) for detecting multiple change points of parameters. Compared to the other distributions, the advantage of GLD is that the GLD family is broad and flexible. The method was applied on fibroblast cancer cell line data, and the change points are successfully located. 


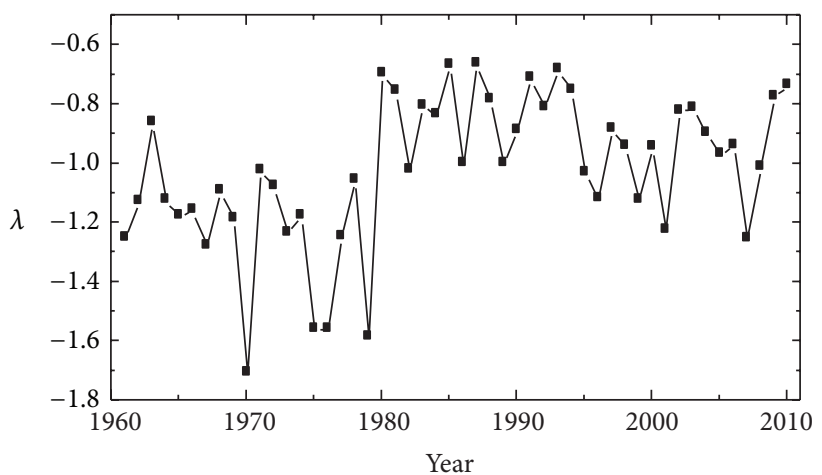

(a)

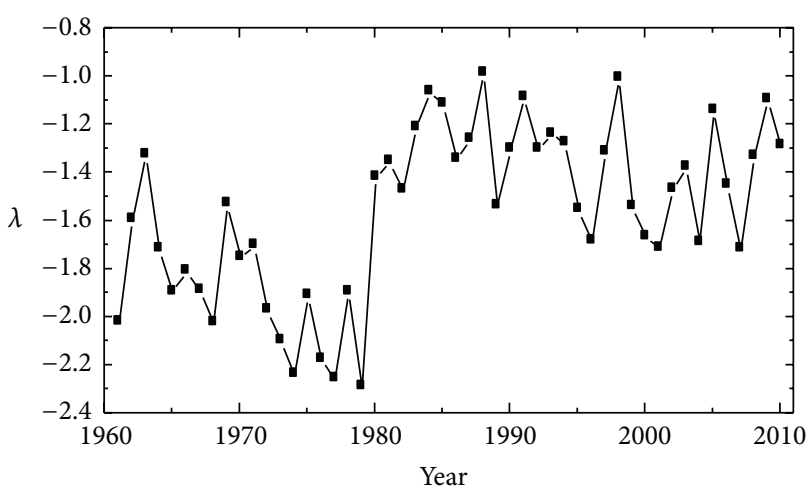

(c)

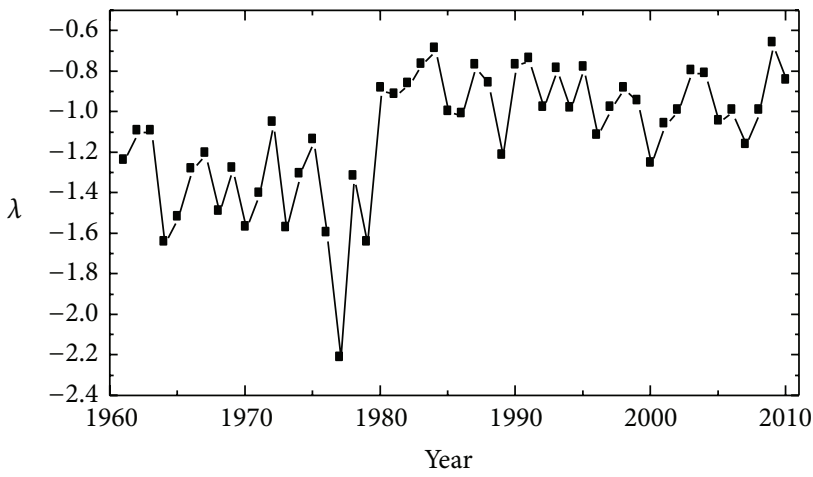

(e)

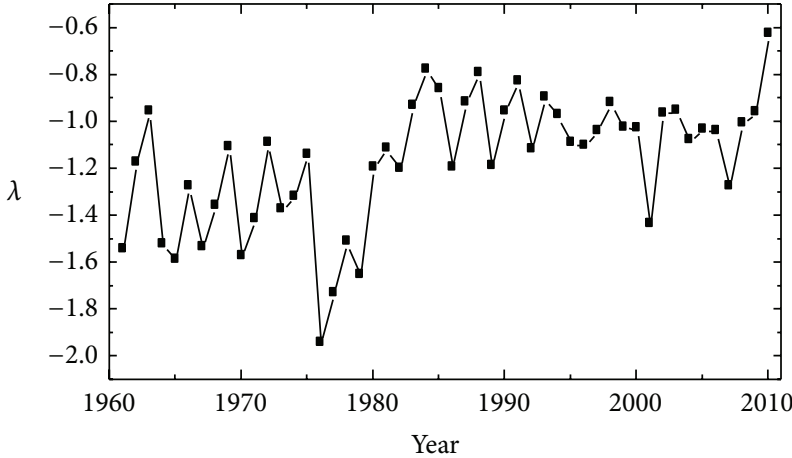

(b)

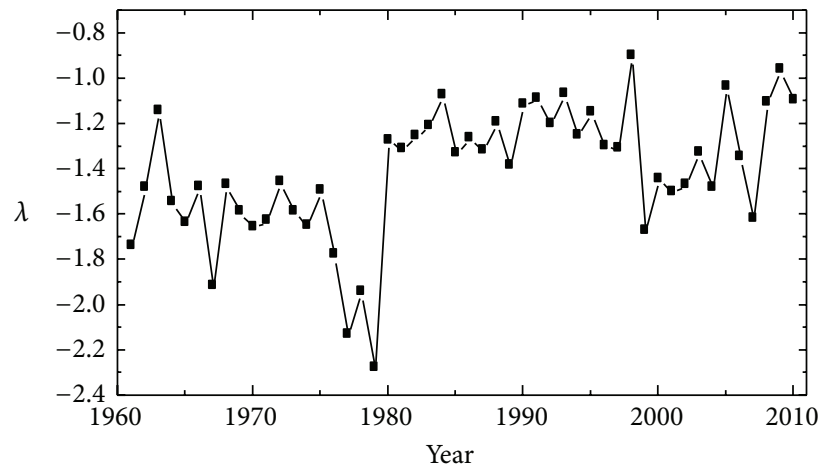

(d)

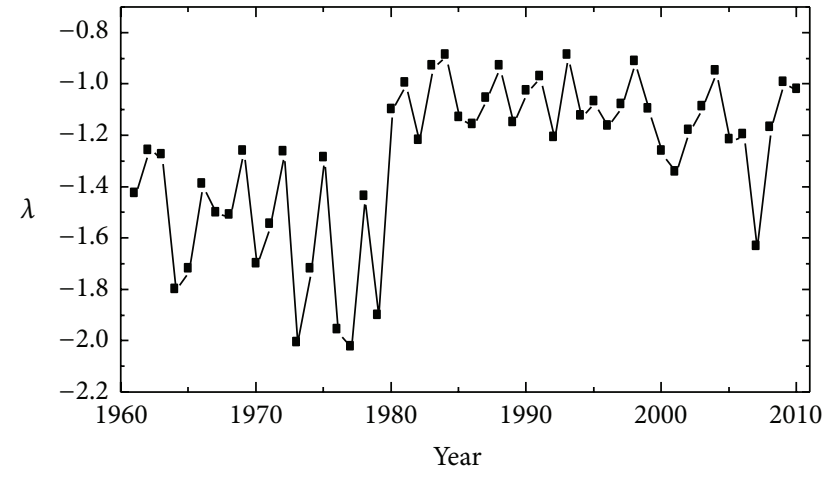

(f)

FIGURE 2: The STP results for daily precipitation records (from 1961 to 2010) in six meteorological observational stations in China, and the sample size of sliding window is 1 year: (a) Tonghe in Heilongjiang Province; (b) Hailun in Heilongjiang Province; (c) Qiqihaer in Heilongjiang Province; (d) Zhalantun in Inner Mongolia; (e) Sunwu in Heilongjiang Province; (f) Nenjiang in Heilongjiang Province [80].

\section{Other Types of Abrupt Detection Methods}

The abrupt variance change can be determined by the change in the second-order statistics of elements. There are few methods to test this type of abrupt change. The DowntonKatz test [84] does not need assumptions for the distribution frequency, but it needs a reference time series without abrupt change. The Nikiforov method [85] can be used to detect an abrupt change in the frequency structure of elements. In using the Nikiforov method, the time series before and after an abrupt change are first introduced into a differential autoregression and moving average model, which can be used to approximately describe the original time series. And then it can detect an abrupt frequency change in the time series by combining with a likelihood ratio test. The Nikiforov test has a strong theoretical basis, but it is only suitable to detect single abrupt change point.

Multivariate analytical methods, such as principal component analysis [43], average standard deviation, and vector autoregression are often used to identify a shift of the spatial pattern of a system. Principal component analysis is a statistical procedure that uses an orthogonal transformation 
to convert a set of observations of possibly correlated variables into a set of values of linearly uncorrelated variables called principal components. The number of principal components is less than or equal to the number of original variables. This transformation is defined in such a way that the first principal component has the largest possible variance. Principal component analysis is the simplest of the true eigenvector-based multivariate analyses and is widely used to identify the coherent patterns of spatial variability. Hare and Mantua [86] applied principal component analysis to study the marine ecosystem in the North Pacific and Bering Strait and found that the abrupt climate change in 1976-1977 had a certain influence on the ecosystem in the North Pacific.

\section{Discussion and Conclusions}

In this paper, we briefly review some advances in the abrupt climate change detection methods in recent years. According to the types of abrupt change, the methods for detecting a state transition in the climate system can be divided into five categories: abrupt mean value change, abrupt variance change, abrupt frequency change, abrupt probability density change, and multivariate analysis. There are relatively numerous research contents on abrupt mean value change and abrupt probability density change. Based on this, we provide a considerably detailed summary. The detection methods for abrupt mean value change mainly have six subcategories, which include the parametric method, the non-parametric method, the cumulative sum method, the Bayesian analysis method, the sequential method, and test algorithms based on regression analysis. The methods for detecting abrupt change in probability density distribution of elements include the coefficient of kurtosis and skewness coefficient and the STP method based on the Box-Cox transformation. There are few testing methods based on an abrupt change in variance, frequency, and multivariate analysis, and the details on the algorithmic methods are not described in the present paper.

In this paper, we mainly describe the methods for climate regime shift detection. These methods have one common shortcoming; that is, the detection results heavily depend on the used sample size. In other words, the detected abrupt change point possesses the characteristics of multiple time scales. The reason for this is that an abrupt change event is often determined according to a statistical significant change of a state variable. So, these detection methods for regime shift are not applicable to the abrupt change in the climate dynamic structure. With the development of nonlinear science, many new methods and new techniques have been presented in recent years, especially for the detection methods for abrupt dynamical change. These methods and techniques are mainly based on the long-range correlation in climate system, the complexity property of time series, and the reconstruction theory of phase space. Different from the detection methods for regime shift, the detection results of these new methods have less dependence on sample size, which are applicable to identify whether there is an abrupt dynamic change in the climate system from single time series.

\section{Conflict of Interests}

The authors declare that there is no conflict of interests regarding the publication of this paper.

\section{Acknowledgments}

This paper is supported by the National Basic Research Program of China (973 Program) (2012CB955902) and the National Natural Science Foundation of China (Grant nos. 41275074, 41475073, and 41175084).

\section{References}

[1] C. B. Fu and Q. Wang, "The definition and detection of the abrupt climate change," Chinese Journal of Atmospheric Sciences, vol. 16, no. 4, pp. 482-493, 1992 (Chinese).

[2] G. X. Wu, Several Questions about Climate Research, Meteorological Press, Beijing, China, 1996, (Chinese).

[3] D. Z. Ye, C. B. Fu, W. J. Dong et al., "Some advance in global change science study," Chinese Journal of Atmospheric Sciences, vol. 27, no. 4, pp. 435-450, 2003 (Chinese).

[4] G.-L. Feng, W.-J. Dong, and X.-J. Jia, "Application of retrospective time integration scheme to the prediction of torrential rain," Chinese Physics, vol. 13, no. 3, pp. 413-422, 2004.

[5] G. L. Feng, W. J. Dong, Z. Q. Gong, W. Hou, S. Q. Wan, and R. Zhi, Nonlinear Theories and Methods on Spatial-Temporal Distribution of the Obserbational Data, Metrological Press, Beijing, China, 2006.

[6] H. Held and T. Kleinen, "Detection of climate system bifurcations by degenerate fingerprinting," Geophysical Research Letters, vol. 31, no. 23, Article ID L23207, 2004.

[7] G.-L. Feng, W.-J. Dong, X.-J. Jia, and H.-X. Cao, “On the dynamics behavour and instability evolution of air-sea oscialltor," Acta Physica Sinica, vol. 51, no. 6, pp. 1184-1185, 2002.

[8] W. P. He, G. L. Feng, Q. Wu, S. Q. Wan, and J. F. Chou, "A new method for abrupt change detection in dynamic structures," Nonlinear Processes in Geophysics, vol. 15, no. 4, pp. 601-606, 2008.

[9] W. P. He, G. L. Feng, Q. Wu, T. He, S. Q. Wan, and J. F. Chou, "A new method for abrupt dynamic change detection of correlated time series," International Journal of Climatology, vol. 32, no. 10, pp. 1604-1614, 2012.

[10] W.-P. He, B.-S. Deng, Q. Wu, W. Zhang, and H.-Y. Cheng, "A new method of detecting abrupt dynamic change based on rescaled range analysis," Acta Physica Sinica, vol. 59, no. 11, pp. 8264-8271, 2010 (Chinese).

[11] E. N. Lorenz, "Nondeterministic theories of climatic change," Quaternary Research, vol. 6, no. 4, pp. 495-507, 1976.

[12] J. G. Charney and J. G. Devore, "Multiple flow equilibria in the atmosphere and blocking," Journal of the Atmospheric Sciences, vol. 36, no. 7, pp. 1205-1216, 1979.

[13] T. J. Crowley and G. R. North, "Abrupt climate change and extinction events in earth history," Science, vol. 240, no. 4855, pp. 996-1002, 1988.

[14] R. B. Alley, J. Marotzke, W. D. Nordhaus et al., "Abrupt climate change," Science, vol. 299, no. 5615, pp. 2005-2010, 2003.

[15] W. S. Broecker, "Does the trigger for abrupt climate change reside in the ocean or in the atmosphere?" Science, vol. 300, no. 5625, pp. 1519-1522, 2003. 
[16] D. Xiao and J. Li, "Main decadal abrupt changes and decadal modes in the global sea surface temperature field," Journal of the Atmospheric Sciences, vol. 31, no. 5, pp. 839-854, 2007 (Chinese).

[17] D. Xiao and J. Li, "Spatial and temporal characteristics of the decadal abrupt changes of global atmosphere-ocean system in 1970s," Journal of Geophysical Research, vol. 112, no. 24, 2007.

[18] D. Xiao, J. Li, and P. Zhao, "Four-dimensional structures and physical process of the decadal abrupt changes of the northern extratropical ocean-atmosphere system in the 1980s," International Journal of Climatology, vol. 32, no. 7, pp. 983-994, 2012.

[19] D. Xiao and J. P. Li, "Mechanism of stratospheric decadal abrupt cooling in the early 1990s as influenced by the Pinatubo eruption," Chinese Science Bulletin, vol. 56, no. 8, pp. 772-780, 2011 (Chinese).

[20] W. P. He, Q. G. Wang, Q. Wu et al., "Comparison of characteristics of moving detrended fluctuation analysis with that of approximate entropy method in detecting abrupt dynamic change," Acta Physica Sinica, vol. 58, no. 4, pp. 2862-2871, 2009 (Chinese).

[21] Q. Q. Liu, W. P. He, and B. Gu, "Application of nonlinear dynamical methods in abrupt climate change detection," Acta Physica Sinica, vol. 64, no. 17, Article ID 179201, 2015.

[22] H. M. Jin, W. P. He, Q. Q. Liu et al., "The applicability of research on moving cut data-approximate entropy on abrupt climate change detection," Theoretical and Applied Climatology, 2015.

[23] K. Z. Zhu, "The enigma of southeast monsoon in China," Journal of Geography, vol. 1, no. 1, pp. 1-27, 1934 (Chinese).

[24] C. W. Tu and T. S. Huang, "The advance and retreats of the summer monsoon in China," Journal of Meteorological Research, vol. 18, pp. 1-20, 1944 (Chinese).

[25] S. Y. Tao and L. X. Chen, "Atmospheric circulation structure over the Asian continent in summer," Journal of Meteorological Research, vol. 28, pp. 234-247, 1957 (Chinese).

[26] D. Z. Ye, S. Y. Tao, and M. C. Li, "The abrupt change of circulation over northern hemisphere during June and October," Journal of Meteorological Research, vol. 29, no. 4, pp. 249-263, 1958 (Chinese).

[27] H. M. Jin, W. P. He, W. Hou, and D.-Q. Zhang, "Effect of different trends on moving cut data-approximate entropy," Acta Physica Sinica, vol. 61, no. 6, Article ID 069201, 2012 (Chinese).

[28] H. M. Jin, W. P. He, W. Zhang et al., "Effect of noises on moving cut data-approximate entropy," Acta Physica Sinica, vol. 61, no. 12, Article ID 129202, 2012, in Chinese.

[29] W. P. He, Q. Q. Liu, Y. D. Jiang, and Y. Lu, "Comparison of performance between rescaled range analysis and rescaled variance analysis in detecting abrupt dynamic change," Chinese Physics B, vol. 24, no. 4, Article ID 049205, 2015.

[30] G.-L. Feng and W.-J. Dong, "Evaluation of the applicability of a retrospective scheme based on comparison with several difference schemes," Chinese Physics, vol. 12, no. 10, pp. 1076-1086, 2003.

[31] G.-L. Feng, W.-J. Dong, and J.-P. Li, “On temporal evolution of precipitation probability of the Yangtze River delta in the last 50 years," Chinese Physics, vol. 13, no. 9, pp. 1582-1587, 2004.

[32] Q.-G. Wang and Z.-P. Zhang, "The research of detecting abrupt climate change with approximate entropy," Acta Physica Sinica, vol. 57, no. 3, pp. 1976-1983, 2008.

[33] W. P. He, S. S. Zhao, Q. Q. Liu, Y. D. Jiang, and B. S. Deng, "Longrange correlation in the drought and flood index from 1470 to 2000 in eastern China," International Journal of Climatology, 2015.
[34] G. Q. Sun, S. L. Wang, Q. Ren, Z. Jin, and Y. Wu, "Effects of time delay and space on herbivore dynamics: linking inducible defenses of plants to herbivore outbreak," Scientific Reports, vol. 5, Article ID 11246, 2015.

[35] Committee on Abrupt Climate Change and National Research Council, Abrupt Climate Change: Inevitable Surprise, National Academy Press, Washington, DC, USA, 2002.

[36] H. Rashid, L. Polyak, and E. M. Thompson, Abrupt Climate Change: Mechanisms, Patterns, and Impacts, American Geophysical Union, Washington, DC, USA, 2013.

[37] W. X. Wu, Y. Hu, and Y. Zhou, "Abrupt climate change and decline of ancient civilization," Journal of Palaeogeography, vol. 11, no. 4, pp. 455-463, 2009 (Chinese).

[38] S. Sahney and M. J. Benton, "Recovery from the most profound mass extinction of all time," Proceedings of the Royal Society B: Biological Sciences, vol. 275, no. 1636, pp. 759-765, 2008.

[39] J. A. Rial, R. A. Pielke Sr., M. Beniston et al., "Nonlinearities, feedbacks and critical thresholds within the earth's climate system," Climatic Change, vol. 65, no. 1-2, pp. 11-38, 2004.

[40] M. Scheffer, J. Bascompte, W. A. Brock et al., "Early-warning signals for critical transitions," Nature, vol. 461, no. 7260, pp. 53-59, 2009.

[41] W. S. Broecker, "Massive iceberg discharges as triggers for global climate change," Nature, vol. 372, no. 6505, pp. 421-424, 1994.

[42] S. N. Rodionov, "A brief overview of the regime shift detection methods," in Large-Scale Disturbances (Regime Shifts) and Recovery in Aquatic Ecosystems: Challenges for Management toward Sustainability, V. Velikova and N. Chipev, Eds., 2005.

[43] N. Mantua, "Methods for detecting regime shifts in large marine ecosystems: a review with approaches applied to North Pacific data," Progress in Oceanography, vol. 60, no. 2-4, pp. 165-182, 2004.

[44] A. Ramanayake and A. K. Gupta, "Change points with linear trend followed by abrupt change for the exponential distribution," Journal of Statistical Computation and Simulation, vol. 72, no. 4, pp. 263-278, 2002.

[45] J. P. Li, J. F. Chou, and J. E. Shi, "Detecting methods on the mean value jump of the climate," Journal of Beijing Meteorological College, vol. 2, no. 16, 1996 (Chinese).

[46] J. P. Li and J. E. Shi, "The detection and analysis of the abrupt change of the global climate during the last 100 years," Chinese Journal of Atmospheric Sciences, vol. 17, pp. 132-140, 1993 (Chinese).

[47] H. B. Mann, "Nonparametric tests against trend," Econometrica, vol. 13, pp. 245-259, 1945.

[48] M. G. Kendall, Rank Correlation Methods, Charles Griffin, London, UK, 1970.

[49] H. B. Mann and D. R. Whitney, "On a test of whether one of two random variables is stochastically larger than the other," Annals of Mathematical Statistics, vol. 18, pp. 50-60, 1947.

[50] Y. Lepage, "A combination of Wilcoxon's and Ansari-Bradley's statistics," Biometrika, vol. 58, no. 1, pp. 213-217, 1971.

[51] J. Morlet, G. Arens, E. Fourgeau, and D. Giard, "Wave propagation and sampling theory-part I: complex signal and scattering in multilayered media," Geophysics, vol. 47, no. 2, pp. 203-221, 1982.

[52] Z. G. Ma and C. B. Fu, "The drought in Northern China during 1951-2004," Chinese Science Bulletin, vol. 51, pp. 2429-2439, 2006 (Chinese). 
[53] W. P. He, T. He, H. Y. Cheng, Z. Wen, and W. Qiong, "A new method to detect abrupt change based on approximate entropy," Acta Physica Sinica, vol. 60, no. 4, Article ID 049202, 2011 (Chinese).

[54] J. P. Li, J. E. Shi, X. C. Lin et al., "Discussion on the efficiency of the Mann-Kendall test to detect the abrupt climatic change," Mathematic Statistics, Supplement, pp. 148-153, 1995 (Chinese).

[55] T. Yonetani, "Discontinuous changes of precipitation in Japan after 1900 detected by the Lepage test," Journal of the Meteorological Society of Japan, vol. 70, no. 1, pp. 95-104, 1992.

[56] T. Yonetani, "Detection of long-term trend, cyclic variation and step-like change by the Lepage test," Journal of the Meteorological Society of Japan, vol. 71, no. 3, pp. 415-418, 1993.

[57] A. Antoniadis and I. Gijbels, "Detecting abrupt changes by wavelet methods," Journal of Nonparametric Statistics, vol. 14, no. 1-2, pp. 7-29, 2002.

[58] E. S. Page, "Continuous inspection schemes," Biometrika, vol. 41, pp. 100-115, 1954.

[59] D. V. Hinkley, "Inference about the change-point from cumulative sum tests," Biometrika, vol. 58, no. 3, pp. 509-523, 1971.

[60] J. M. Lucas and R. B. Crosier, "Fast initial response for CUSUM quality-control schemes: give your CUSUM a head start," Technometrics, vol. 24, no. 3, pp. 199-205, 1982.

[61] G. V. Moustakides, "Optimality of the CUSUM procedure in continuous time," The Annals of Statistics, vol. 32, no. 1, pp. 302315,2004

[62] V. Janssen, "Detection of abrupt baseline length changes using cumulative sums," Journal of Applied Geodesy, vol. 3, no. 2, pp. 89-96, 2009.

[63] J. B. Elsner, T. Jagger, and X.-F. Niu, "Changes in the rates of North Atlantic major hurricane activity during the 20th century," Geophysical Research Letters, vol. 27, no. 12, pp. 1743-1746, 2000.

[64] C. Andrieu, N. De Freitas, A. Doucet, and M. I. Jordan, "An introduction to MCMC for machine learning," Machine Learning, vol. 50, no. 1-2, pp. 5-43, 2003.

[65] P.-S. Chu and X. Zhao, "Bayesian change-point analysis of tropical cyclone activity: the central North Pacific case," Journal of Climate, vol. 17, no. 24, pp. 4893-4901, 2004.

[66] J. B. Elsner, X. F. Niu, and T. H. Jagger, "Detecting shifts in hurricane rates using a Markov Chain Monte Carlo approach," Journal of Climate, vol. 17, no. 13, pp. 2652-2666, 2004.

[67] S. N. Rodionov, "A sequential algorithm for testing climate regime shifts," Geophysical Research Letters, vol. 31, no. 9, Article ID L09204, 2004.

[68] S. Rodionov and J. E. Overland, "Application of a sequential regime shift detection method to the Bering Sea ecosystem," ICES Journal of Marine Science, vol. 62, no. 3, pp. 328-332, 2005.

[69] P. C. Reid, R. E. Hari, G. Beaurand et al., "Global impacts of the regime shift," Global Change Biology, 2015.

[70] P. Bernaola-Galvan, P. C. Ivanov, L. A. N. Amaral, and H. E. Stanley, "Scale invariance in the nonstationarity of human heart rate," Physical Review Letters, vol. 87, Article ID 168105, 2001.

[71] G.-L. Feng, Z.-Q. Gong, W.-J. Dong, and J.-P. Li, "Abrupt climate change detection based on heuristic segmentation algorithm," Acta Physica Sinica, vol. 54, no. 11, pp. 5494-5499, 2005 (Chinese).

[72] Z.-Q. Gong, G.-L. Feng, S.-Q. Wan, and J.-P. Li, "Analysis of features of climate change of Huabei area and the global climate change based on heuristic segmentation algorithm," Acta Physica Sinica, vol. 55, no. 1, pp. 477-484, 2006 (Chinese).
[73] D. V. Hinkley, "Inference about the intersection in two-phase regression," Biometrika, vol. 56, no. 3, pp. 495-504, 1969.

[74] D. V. Hinkley, "Inference in two phase regression," The Journal of the American Statistical Association, vol. 66, no. 336, pp. 736743, 1971.

[75] R. Lund and J. Reeves, "Detection of undocumented change points: a revision of the two-phase regression model," Journal of Climate, vol. 15, no. 17, pp. 2547-2554, 2002.

[76] X. L. Wang, "Comments on: detection of undocumented changepoints: a revision of the two-phase regression model," Journal of Climate, vol. 16, no. 20, pp. 3383-3385, 2003.

[77] D. L. Rudnick and R. E. Davis, "Red noise and regime shifts," Deep Sea Research Part I: Oceanographic Research Papers, vol. 50, no. 6, pp. 691-699, 2003.

[78] S. N. Rodionov, "Use of prewhitening in climate regime shift detection," Geophysical Research Letters, vol. 33, no. 12, 2006.

[79] H. Y. Cheng, W. P. He, T. He et al., "A new approach to abrupt change detection based on change of probability density distribution," Acta Physica Sinica, vol. 61, no. 3, Article ID 039201, 2012 (Chinese).

[80] W. He, S. Wan, Y. Jiang et al., "Detecting abrupt change on the basis of skewness: numerical tests and applications," International Journal of Climatology, vol. 33, no. 12, pp. 2713-2727, 2013.

[81] G. E. P. Box and D. R. Cox, "An analysis of transformations," Journal of the Royal Statistical Society-Series B: Methodological, vol. 26, pp. 211-252, 1964.

[82] G. E. P. Box and D. R. Cox, "An analysis of transformation revisited, rebutted," Journal of the American Statistical Association, vol. 77, no. 377, pp. 209-210, 1982.

[83] W. Ning and A. K. Gupta, "Change point analysis for generalized lambda distribution," Communications in Statistics-Simulation and Computation, vol. 38, no. 9, pp. 1789-1802, 2009.

[84] T. R. Karl and C. N. Williams Jr., "An approach to adjusting climatological time series for discontinuous inhomogeneities," Journal of Climate \& Applied Meteorology, vol. 26, no. 12, pp. 1744-1763, 1987.

[85] M. Basseville and I. V. Nikiforov, Detection of Abrupt Change: Theory and Application, Prentice-Hall, Englewood Cliffs, NJ, USA, 1993.

[86] S. R. Hare and N. J. Mantua, "Empirical evidence for North Pacific regime shifts in 1977 and 1989," Progress in Oceanography, vol. 47, no. 2-4, pp. 103-145, 2000. 


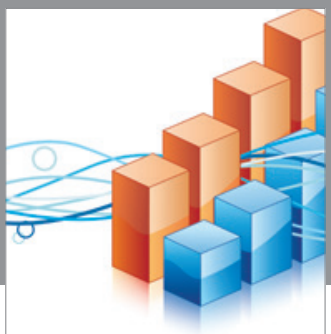

Advances in

Operations Research

vatem alat4

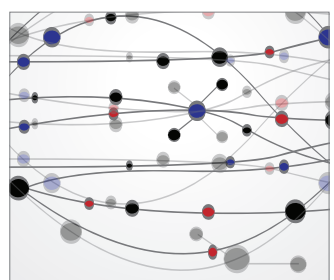

\section{The Scientific} World Journal
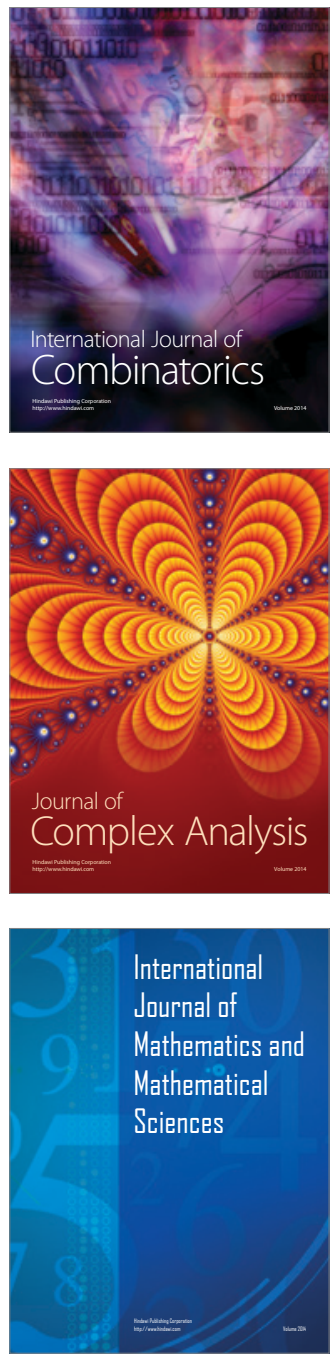
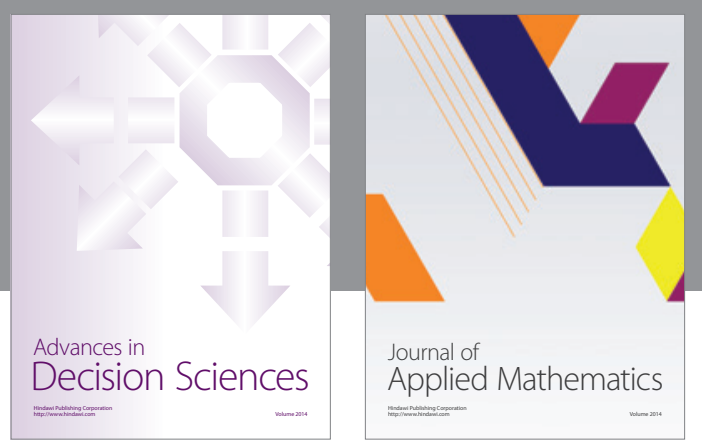

Algebra

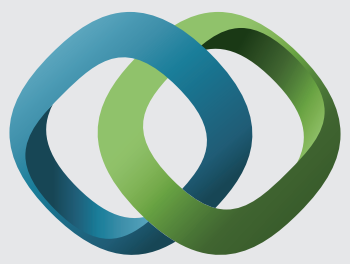

\section{Hindawi}

Submit your manuscripts at

http://www.hindawi.com
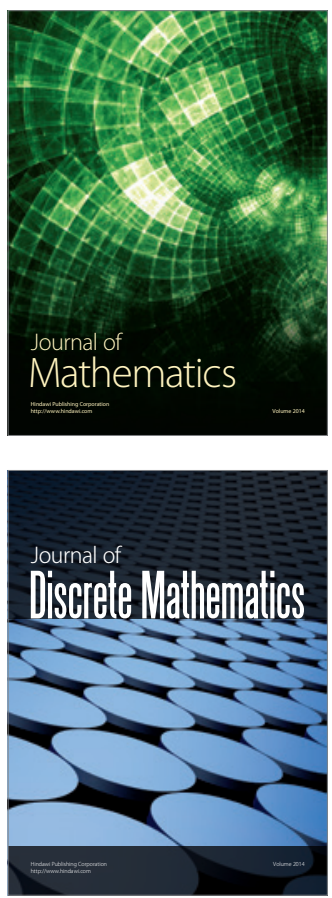

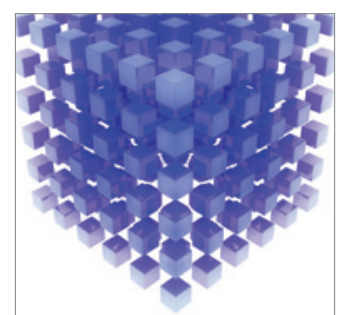

Mathematical Problems in Engineering
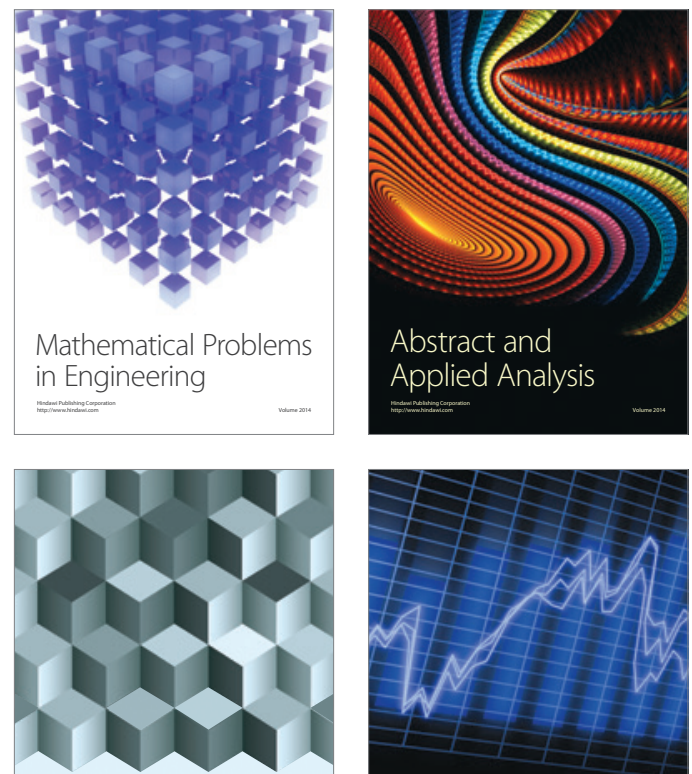

Journal of

Function Spaces

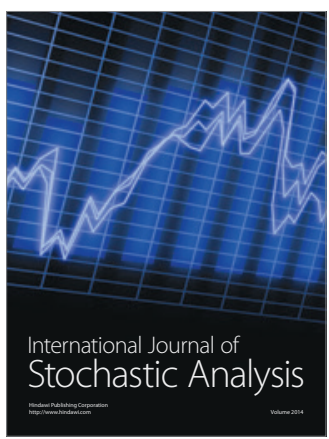

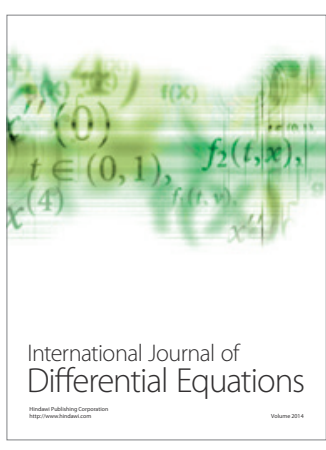
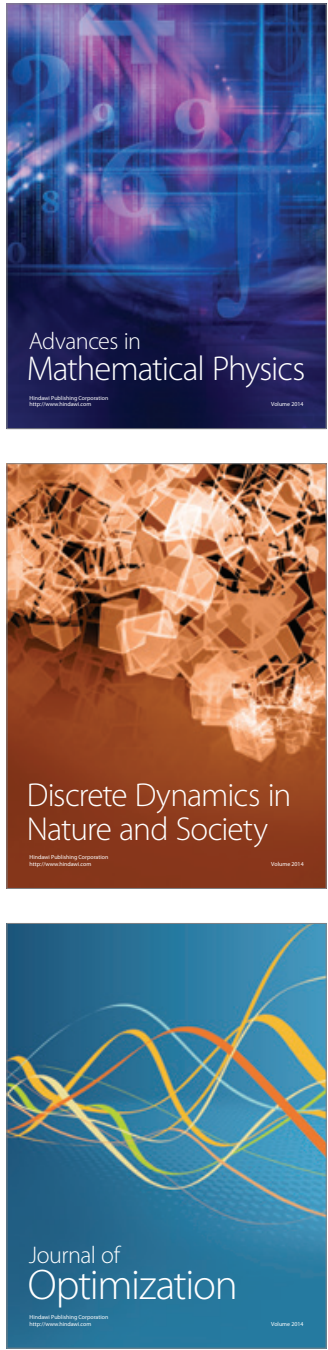